\title{
DESCRIBING THE ASYMMETRIES IN EXTRAGALACTIC
}

\section{RADIO SOURCES}

\author{
STANISŁAW RYŚ \\ Astronomical Observatory of the Jagiellonian University \\ PL - 30244 KRAKÓW, ul. Orla 171, POLAND, \\ E-mail: strys@oa.uj.edu.pl
}

As an ideally symmetrical regime we assume that central engine ejects the same volumes of radio emitting matter - hereafter called 'plasmons' - into two opposite directions. The plasmons have the identical velocities and evolution and are small. We assume that each plasmon on one side has its corresponding twin on the opposite side of the structure. Since in fact observations do not reveal any symmetrical structures we must include into our model some mechanism to reproduce an asymmetry. It is our assumption that such asymmetry is due to the fact that the observer sees the plasmons at their different evolutionary ages and that their velocity vectors have different directions. We are looking for the functions which describe differences between the twins. These functions give us differences in brightness and expected positions of the twins and may be used for interchanging their elements. This exchange leads to obtaining new structure. If this new structure is identical to the original one, then the functions correctly describe the differences between the two sides of the structure (Paper 1).

The relativistic flip-flop model described in Paper 2 was tested by applying it to the description of the asymmetry in 33 radio structures selected from the survey by Machalski and Condon (1983). The parameters which represent spatial interval between the switching of activities of the central engine $(B)$ and the relative life-time $(\tau)$ are reasonably correlated (correlation coefficient $\rho=0.59$ ). Taking into account this value of the correlation coefficient I suggest a linear dependence $B \sim \log (\tau)$ (Fig. 1). If we assume that all sources have the same physical values for twin plasmon delay and characteristic time scale of plasmon shining rate, we obtain sincle interpretation of this dependence as follows: Older structures have smaller parameters $B$ and $\tau$ than younger structures. 


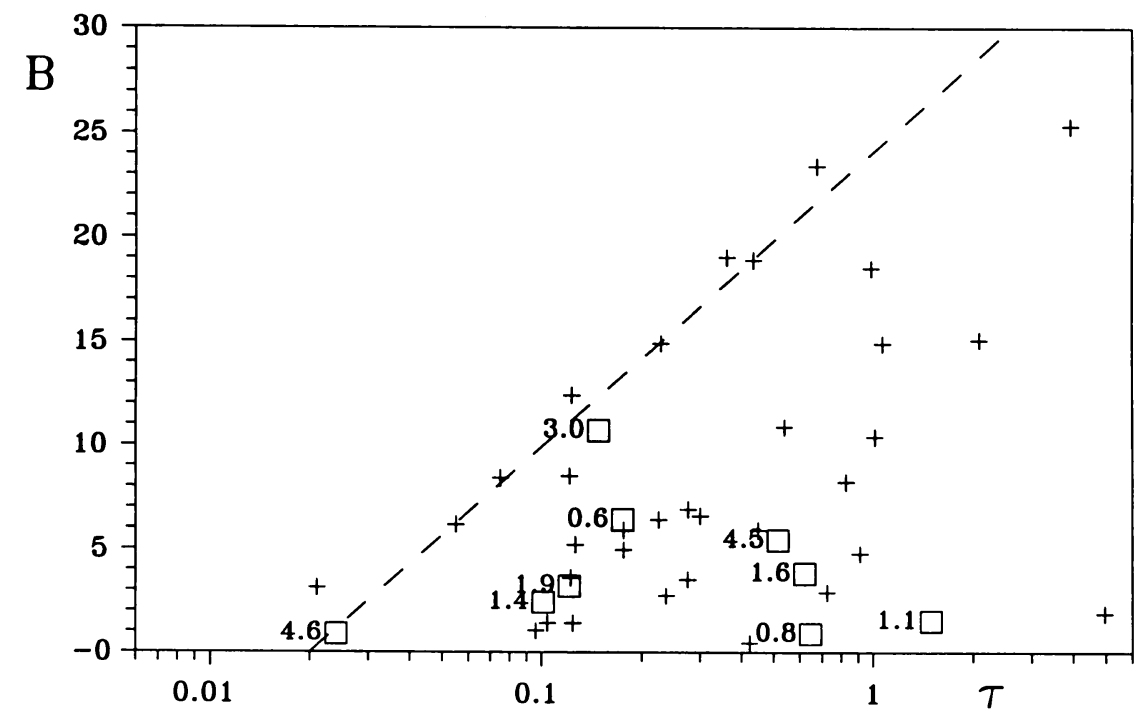

Figure 1. The relationship between $B$ flip-flop parameter and the relative life-time in the model. The dotted line $(B=-0.17+8.54 \log (\tau))$ is an example of the evolutionary path of radio structure. $A \& L$ sources are marked by $\square$ and numbers describes their age (in unit of $10^{7}$ years).

In the sample investigated in Paper 2 there are no structures for which the age could be estimated from other independent investigations. Therefore I added nine sources from Alexander \& Leahy (1987) sample (hereafter $A \& L$ ) marked by rectangles in Figure 1 . The ages of radio sources determined by $A \& L$ give the sequence without agreement of ages determined by parameters $B$ and $\tau$. This result we can interpret in two ways:

- the simple interpretation is not valid (we need more than one physical model for description of asymmetries - Chyży 1994);

- the dynamical ages of radio structures are different from the values approximated from $A \& L$ (see J. Eilek in this proceedings).

Acknowledgments. I would like to express my gratitude to the IAU Secretary for travel grant. I thank my colleagues K.Maślanka and K. Chyży for fruitful discussion. The work was supported by KBN grant no. 578/P03/95/09.

\section{References}

Alexander P., Leahy J.P., (1987) Astron.Astrophys., 225,1

Chyży K.T., (1994), in "AGN Across the Electromagnetic Spectrum" ed. Courvoisier T.J.-L., Blecha A.R., (Kluwer) p.501

Machalski J., Condon J.J., (1983) Astron.J., 88,143

Ryś S.: (1994) Astron.Astrophys., 281,15 (Paper 1)

Ryś S. (1995) submitted to Astron.Astrophys. (Paper 2) 